\section{Invasive aspergillosis complicated by subclavian artery occlusion and costal osteomyelitis after autologous bone} marrow transplantation

\author{
L Th Vlasveld, J F M Delemarre, \\ J H Beynen, $S$ Rodenhuis
}

\begin{abstract}
A 47 year old woman presented with invasive pulmonary aspergillosis complicated by subclavian artery occlusion and rib fracture due to fungal osteomyelitis. This followed persistent pancytopenia resulting from autologous bone marrow transplantation for metastatic breast cancer.
\end{abstract}

Department of Medical Oncology cases. ${ }^{1}$ The lung is the primary site of L Th Vlasveld $S$ Rodenhuis

Department of Pathology

J F M Delemarre

Department of

Pharmacy

J H Beynen

Netherlands Cancer

Institute, Plesmanlaan

$121,1066 \mathrm{CX}$

Amsterdam, The

Netherlands

Reprint requests to:

Dr Vlasveld

Accepted 12 August 1991

Invasive aspergillosis complicates autologous bone marrow transplantation in $3-12 \%$ of infection in $90 \%$, where it causes rapidly necrotising bronchopneumonia. Pulmonary haemorrhagic infarction and haematogenous dissemination are sequelae of the vasoinvasive properties of the fungus. ${ }^{2}$ Clinically apparen local extension to contiguous structures is uncommon.

\section{Case report}

A 47 year old woman was treated with high

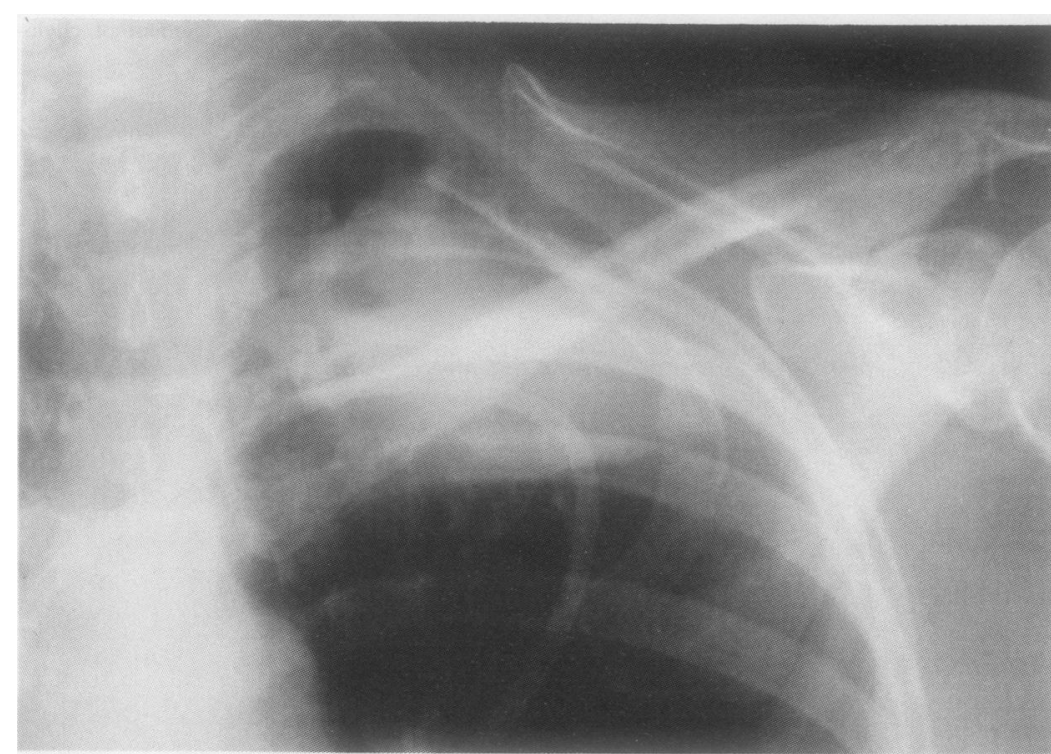

Detail of a chest radiograph showing the cavitating pulmonary aspergillosis with costal osteomyelitis complicated by a fracture of the first rib with associated pneumothorax and subcutaneous emphysema. dose melphalan and mitoxantrone followed by reinfusion of autologous bone marrow in an attempt to consolidate a clinically complete remission of metastatic breast cancer after conventional chemotherapy. Bone marrow engraftment was delayed and was followed by secondary take failure, which persisted until her death over four months after transplantation.

She developed persistent fever, despite broad spectrum antibiotics, and an intractable dry cough, dyspnoea, migrating pleuritic friction rubs, and pulmonary infiltrates on the chest radiograph. Intravenous amphotericin B $(0.5 \mathrm{mg} / \mathrm{kg})$ was therefore started on day 20 after transplantation. In the sixth week peripheral arterial pulsations in the left arm were found to be absent without signs of hypoperfusion. Digital subtraction angiography showed occlusion of the left subclavian artery. Aspiration from a cavitating infiltrate in the left upper lobe on day 48 yielded growth of an Aspergillus species. Oral itraconazole was started, at a dose of $200 \mathrm{mg}$ twice daily. Because of persistent fever, fungal colonisation of the alimentary and respiratory tracts and progression of the pulmonary infiltrates, itraconazole was discontinued and intravenous amphotericin B $(0.75 \mathrm{mg} / \mathrm{kg})$ was resumed on day 63 . On day 96 the patient complained of severe pain in the left upper chest wall, which appeared to be caused by a fractured rib as a result of costal osteomyelitis (figure). Because of deterioration of renal function, standard amphotericin B was replaced by liposomal amphotericin B ( $3 \mathrm{mg} / \mathrm{kg}$; Vestar, San Dimas, California) on day 101 and renal function returned to normal.

The patient died 118 days after bone marrow reinfusion as a result of refractory Pseudomonas maltophila septicaemia. By then she had had antifungal treatment for $\mathbf{9 8}$ days (total dose amphotericin B $3985 \mathrm{mg}$ and itraconazole $6000 \mathrm{mg}$ ).

At necropsy a necrotic mass with a diameter of $5 \mathrm{~cm}$ in the left upper lobe was contiguous with the ribs and extended to the left supraclavicular region, with occlusion and obliteration of the subclavian artery and vein. Aspergillus was found throughout the necrotic mass, in the affected ribs, and in the wall of the subclavian vessels. Metastatic breast cancer was seen in the right breast, regional lymph nodes, skin, and bone marrow.

\section{Discussion}

Although vasoinvasive growth with subsequent thrombosis is the pathological hallmark of invasive aspergillosis, occlusion of large vessels has only been reported occasionally. ${ }^{2-4}$ This condition has usually been rapidly fatal and in most patients Aspergillus was identified as the cause of the occlusion only at necropsy. In some cases the blood vessel was obstructed as a result of fungal embolism, whereas in others there was direct vasoinvasive growth from contiguous infected tissue. Aspergillus osteomyelitis is uncommon and is caused by haematogenous fungal spread or by 
local invasion from contiguous infected tissue, an artificial prosthesis, or open wounds..$^{5-7}$ Nine cases of osteomyelitis due to local invasion from adjacent pulmonary aspergillosis have been reported.

Although amphotericin B is still the drug of choice for invasive aspergillosis, the disease progressed during treatment in our patient, with persistent neutropenia. In view of the lack of convincing clinical data on the therapeutic advantage of combination treatment, we refrained from adding 5-fluorocytosine or rifampicin as our patient had pancytopenia and jaundice. ${ }^{89}$ The therapeutic role of liposome encapsulated amphotericin B and the new oral triazole derivates such as itraconazole in neutropenic patients is not yet well established. ${ }^{810}$

Although cure of Aspergillus osteomyelitis may be achieved with either amphotericin B or surgery alone a combination of antifungal agents and surgical debridement has been advocated to treat Aspergillus osteomyelitis. ${ }^{56}$ Because of the rapidly fatal outcome of occlusion of great vessels associated with fungus, data on the best therapeutic strategy are not available. Infected vascular grafts may be treated successfully by surgical resection in combination with systemic antifungal treatment. ${ }^{7}$ In our patient invasive procedures such as thoracoplasty or intracavitary drug administration were not considered feasible because of her haematological condition and the local extension to the ribs. ${ }^{112}$

Although invasive aspergillosis may be cured with early and aggressive amphotericin B treatment in many neutropenic patients, prevention remains very important. ${ }^{9}$ For that purpose prophylaxis with systemic low dose amphotericin B and the use of growth factors to shorten the neutropenic period may be helpful. ${ }^{13}$

1 Kirk JL, Greenfield RA, Slease B, Epstein RB. Analysis of early infectious complications after autologous bone marrow transplantation. Cancer 1988;62:2445-50.

2 Rinaldi MG. Invasive aspergillosis. Rev Infect Dis 1983; 5:1061-77.

3 Gartenberg G, Einstein K, Jagirdar J. Superior vena cava syndrome caused by invasive aspergillosis. Chest 1978; 74:671-2.

4 Byard RW, Jimenez CL, Carpenter BF, Hsu E. Aspergillusrelated aortic thrombosis. Can Med Ass J 1987;136:155-6.

5 Tack KJ, Rhame FS, Brown B, Thompson RC. Aspergillus osteomyelitis-report of four cases and review of the literature. Am J Med 1982;73:295-300.

6 Barnwell PA, Jelsma LF, Raff MJ. Aspergillus osteomyelitis-report of a case and review of the literature. Diagn Microbiol Infect Dis 1985;3:515-9.

7 Anderson J, Kron IL. Treatment of aspergillus infection of the proximal aortic prosthetic graft with associated vertebral osteomyelitis. J Vasc Surg 1984;1:579-81.

8 Denning DW, Stevens DA. Antifungal and surgical treatment of invasive aspergillosis: review of 2,121 published cases. Rev Infect Dis 1990;12:1147-201.

9 Burch PA, Karp JE, Merz WG, Kuhlman JE, Fishman EK. Favourable outcome of invasive aspergillosis in patients with acute leukemia. J Clin Oncol 1987;5:1985-93.

10 Davey PG. New antiviral and antifungal drugs. BMJ 1990; 300:793-8.

11 Al-Zeerah $M$, Jeyasingham $\mathrm{K}$. Limited thoracoplasty in the management of complicated pulmonary aspergillomas. Thorax 1989;44:1027-30.

12 Shapiro MJ, Albelda SM, Maycock RL, McLean GK. Severe hemoptysis associated with pulmonary aspergilloma: percutaneous intracavitary treatment Chest 1988;94 1225-31.

13 O'Donnell MR, Schmidt GM, Tegtmeier B, et al. Propylactic low dose amphotericin $B$ (AM-B) decreases systemic fungal infection (SFI) in allogenic bone marrow transplant (BMT) recipients [abstract]. Blood 1990;76 (suppl plant (BM. 